\title{
LOOP BASED CLASSIFICATION OF PLANAR SCISSOR LINKAGES
}

\author{
Gökhan Kiper ${ }^{\mathrm{a} *}$, Koray Korkmaz ${ }^{\mathrm{b}}$, Şebnem Gürb ${ }^{\mathrm{b}}$, Müjde YAR Uncu ${ }^{\mathrm{b}}$, Feray Maden ${ }^{\mathrm{c}}$, \\ Yenal Akgün ${ }^{c}$, Cevahir Karagöza \\ ${ }^{a}$ Department of Mechanical Engineering, Izmir Institute of Technology, 35430 İzmir, Turkey \\ ${ }^{\mathrm{b}}$ Department of Architecture, İzmir Institute of Technology, 35430 İmir, Turkey \\ ${ }^{\mathrm{c} D}$ Department of Architecture, Yaşar University, 35100, İzmir, Turkey
}

*Corresponding author: G. Kiper: gokhankiper@iyte.edu.tr, ORCID: 0000-0001-8793-724X

\begin{abstract}
Scissor linkages have been used for several applications since ancient Greeks and Romans. In addition to simple scissor linkages with straight rods, linkages with angulated elements have been introduced in the last decades. In the related literature, two methods have been used to design scissor linkages, one of which is based on scissor elements, and the other is based on assembling loops. This study presents a systematic classification of scissor linkages as assemblies of rhombus, kite, dart, parallelogram and anti-parallelogram loops using frieze patterns and long-short diagonal connections. After the loops are replicated along a curve as a pattern, the linkages are obtained by selection of proper common link sections for adjacent loops. The resulting linkages are analyzed for their motions and they are classified as realizing scaling deployable, angular deployable or transformable motion. Some of the linkages obtained are novel. Totally 10 scalable deployable, 1 angular deployable and 8 transformable scissor linkages are listed. Designers in architecture and engineering can use this list of linkages as a library of scissor linkage topologies.
\end{abstract}

Keywords: deployable structures; planar scissor linkages, loop based design, topological classification

\section{Introduction}

Scissor linkages have been used for deployment for thousands of years. Folding tripods with a pair of scissor links on each side have been used by the Greeks and Romans starting from the times before the Common Era [1]. Today, many deployable structures comprising scissor-like elements (SLEs) are used in wide range of applications such as household goods, lifts, architecture and outer-space structures. The academic studies on the use of scissor linkages as deployable structures are dated back to 1960s, where Piñero [2] developed a movable theatre composed of rigid bars and cables. Using the principles of SLEs, Piñero [3-4] also proposed several structures for pavilions and retractable domes. Piñero's designs require to use some additional elements to lock the system and to provide the necessary stabilization after folding. The disadvantages that are inherent in his designs led other researchers to investigate the scissor structures that do not require additional members for stabilization. Zeigler [5] developed a self-supported dome structure and Clarke [6] designed a deployable hemispherical dome composed of a novel spatial unit. Although specific configurations of his structure seemed to work fairly well, it allowed only limited geometric shapes and few applications.

The research on deployable structures was expanded by Escrig [7-8] who first presented the geometric conditions for deployability of scissor linkages composed of translational and polar SLEs. Escrig also developed spherical grid structures and different types of deployable scissor structures including quadrilateral expandable umbrella, deployable polyhedral structure and compactly folded cylindrical and geodesic structures [9-11]. A large-scale deployable scissor structure proposed by Escrig [12] was built in Seville to cover the swimming pool in San Pablo Sports Center.

Chuck Hoberman [13] made a remarkable invention on scissor structures with the angulated scissor element (Fig. 1a). The discovery of this element extended the range of application of single degrees-of-freedom (DOF) scissor structures since it allows the structure to radially deploy from a center to the perimeter. Hoberman [14] created impressive examples of scissor structures by using the angulated elements. Expanding Geodesic Dome, Hoberman Arch, Expanding Sphere, Expanding Icosahedron, Iris Dome and Expanding Helicoid are some of his interesting designs. You and Pellegrino [15] investigated the conditions on the link lengths for which angulated elements subtend a constant angle and found two conditions leading to two types of generalized angulated elements (GAEs) (Fig. 1b, c). Hoberman's pioneering idea on the angulated element led Kassabian, You and Pellegrino [16] make 
further progress on scissor structures and they discovered multi-angulated elements which comprise links with more than three joints. Based on the principles of multi-angulated elements, they developed deployable scissor structures with circular and elliptical layouts. Al Khayar and Lalvani [17] studied the applications of angulated elements to polygonal hyperboloids and proposed many types of deployable hyperboloids by using regular and semi-regular tessellations (tessellations of a single type or several types of regular polygons, respectively).

a)

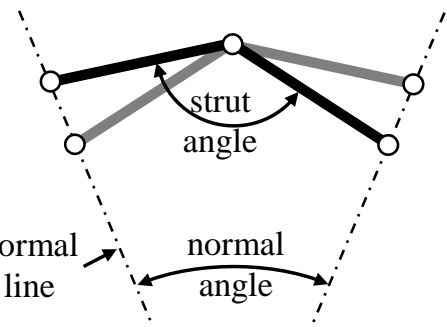

b)

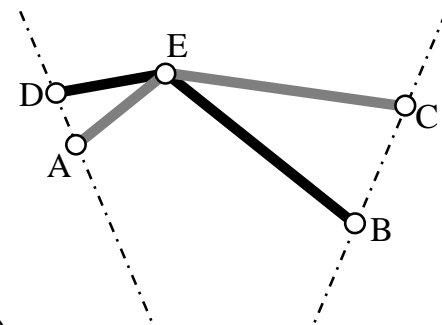

c)

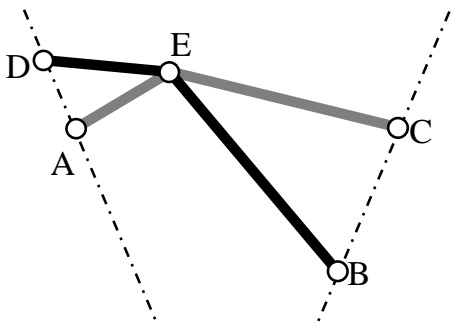

Figure 1 a) An angulated element pair with equal link lengths and kink angles, b) An equilateral GAE: $|\mathrm{AE}|=|\mathrm{DE}|$, $|\mathrm{BE}|=|\mathrm{CE}|, \angle \mathrm{AEC} \neq \angle \mathrm{BED}, \mathrm{c}) \mathrm{A}$ similar GAE: $|\mathrm{AE}| /|\mathrm{DE}|=|\mathrm{CE}| /|\mathrm{BE}|, \angle \mathrm{AEC}=\angle \mathrm{BED}$

Gantes [18] systematically investigated "snap-through" effect (sudden change of buckling mode) of the scissor structures that occurs at intermediate geometric configurations due to the geometric incompatibilities between the member lengths. He developed geometric design methodologies and determined deployability conditions for different types of scissor structures in order to achieve stable and stress-free states of such systems [19]. Langbecker [20] studied the foldability conditions of SLEs and presented a systematic method for the kinematic analysis of the scissor structures. Using compatible translational SLEs, he also proposed foldable singly-curved barrel vaults (a structure obtained by extruding a curve) and doubly-curved synclastic structures (a structure with a surface curved toward the same side in all directions) [21]. Kokawa [22-23] designed an expandable arch composed of scissor pairs and cables and also a retractable loop-dome consisting of 3D multi-angulated SLEs in lamella arrangement such that the SLEs' hinge points are located on the intersections of a sphere with inclined planes.

Van Mele [24] proposed a deployable barrel vault structure by using scissor arches composed of angulated elements to cover a tennis arena. Rather than using a single scissor arch that is pinned at one end, the scissor arch is cut in half and two halves are pinned to the spectator area that are connected at a central hinge in the closed configuration. Rippmann [25] developed a scissor unit that has various intermediate hinge points. By this means, he proposed a structure that can constitute different geometric shapes by switching the locations of the hinge points in the basic scissor unit. Although it seems that the structure provides form flexibility, in fact, the scissor units have to be dismantled first and then connected again to obtain the desired shape configurations.

Petrova [26] investigated doubly-curved structures with arbitrary surfaces. She studied all principle kind of curvatures to design more arbitrary surfaces and free forms. She developed a design methodology to generate arbitrary doubly-curved translational surfaces. By using rhombic scissor units, she designed many anticlastic structures (a structure with a surface which has opposite curvatures at a certain points) in which the shape and curvature of the surface can be set arbitrarily. Besides, she proved the feasibility of such structures and developed prototypes.

Akgün [27] developed three types of modified SLEs in which additional revolute joints have been added on various locations of a bar. Based on the modified SLEs, he introduced new adaptable scissor structures that are capable of transforming from flat geometries to various curved shapes without changing the span (width) of the structure. Akgün [27] also proposed an adaptive roof structure for an exhibition hall by using six scissor arches. The proposed structure provides wide range of form flexibility by allowing the transformation from arch shapes to various curved ones. Another transformable structure developed by Akgün is a 4-DoF spatial scissor structure that is composed of 25 spatial SLEs, 4 modified spatial SLEs, 20 hybrid spatial SLEs and 8 special SLEs [28]. 
Roovers et al. [29] searched for new geometric shapes to reveal the potential applications of angulated elements to innovative geometries. Rather than using simply curved surfaces such as cylindrical or spherical shapes, they developed a design method that any arbitrary continuous surface can be converted into an angulated scissor grid. After testing various surfaces and families of surfaces, they developed a single DOF deployable catenoid structure.

Zhang et al. [30] developed a methodology for designing scissor linkages for the transformation of a curve shape to another. Bouleau and Guscetti [31] utilized a simplified version of Zhang et al.'s [30] method to design a transformable bridge which can transform form a flat configuration to a curved configuration.

In his first patent about angulated elements, Hoberman [13] described how identical angulated elements are paired to form angulated scissor-pairs. In the patent, the angle of an angulated element is called a "strut angle", the line connecting the left or right terminals of a pair of elements is called a "normal line" and the angle between two normal lines is called a "normal angle" (Fig. 1a). Although Hoberman [13] describes an angulated scissor-pair as a module, he calls his mechanisms as "loop assemblies" implying that he constructs the mechanisms by assembling loops (in this case rhombuses). Also, the "normal line"s are normal to the curve to be approximated.

Later on, in a lecture at MIT, Hoberman [32] described his construction of expanding polygons as an assembly of "hinged rhombs" and calls the "normal lines" as "perpendicular bisectors" of polygonal sides. Although it is not mentioned in the patent, this latter study shows that Hoberman assembles rhombus loops to obtain his linkages. Similar deployable structures are also issued by Liao and $\mathrm{Li}$ [33] and Kiper and Söylemez [34], but their procedures are not based on assembling loops.

Bai et al. [35] assembled rhombus, parallelogram, kite and general quadrilateral loops for polygon scaling, where they discuss different ways to assemble these loops as well. Yar et al. [36] used kite and dart loops and Gür et al. [37-38] used anti-parallelogram loops to obtain planar scissor linkages, some of which are deployable, whereas some are transformable. Karagöz [39] presented examples of design formulations for several deployable and transformable scissor linkages using the loop assembly method. Maden et al. [40] developed a dynamic shelter structure based on S-shaped loop assembly which can transform from a flat configuration to both S-shaped and reversed S-shaped forms.

Two main methods to design scissor structures are: 1) assembling scissor units composed of two ternary links, and 2) assembling 4-bar loops. A review of scissor structures based on assembly of scissor units is given by Maden, Korkmaz and Akgün [41]. Recently, Maden et al. [42] published a review paper on scissor structures which examines the literature on both unit-based and loop-based design methods and summarizes the different terminologies used in the literature. The paper also proposes a new classification for planar scissor structures according to their motions: 1) scaling/dilation type deployable structures (the angles are preserved but the form is scaled), 2) angular deployable structures (the angles are varied in an arc form), 3) other transformable structures (Fig. 2). Scaling deployment contains linear deployment along a line and radial deployment (enlarging circular arc) as special cases. This classification for the transformation types is followed in this study as well.

This study classifies and systematically analyzes assembly of suitable loops for planar deployable structures comprising SLEs. According to the authors' best knowledge, this is the first full classification of scissor mechanisms considered as loop assemblies. As most of the assemblies are noted in the literature, there are several novel assemblies listed in this paper. In Section 2, the loops are introduced. In Section 3, possible ways of assembling different loops are presented and the geometric transformation properties of the assemblies are examined. Section 4 concludes the paper. 


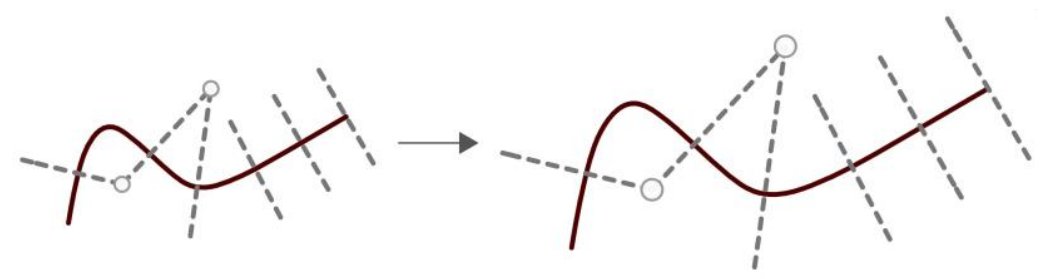

(a)

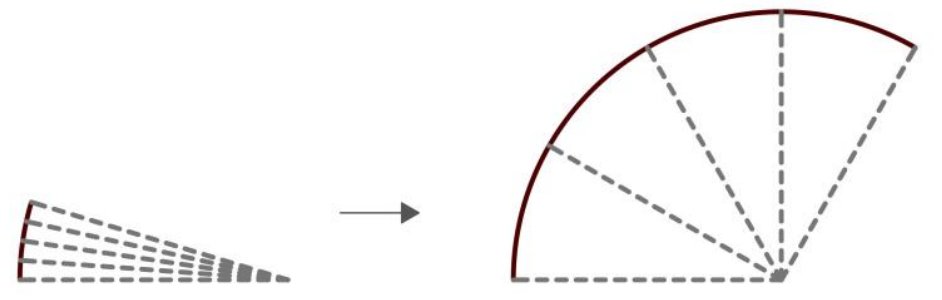

(b)

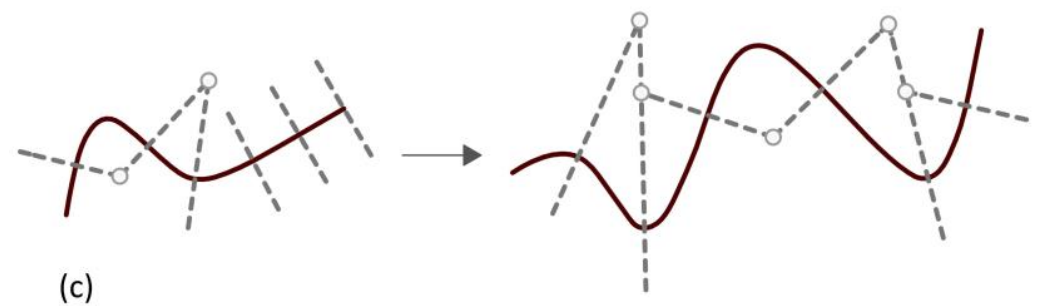

Figure 2 Deployable vs. transformable motion: a) scaling/dilation type deployable; b) angular deployable; c) transformable [42]

\section{Loops}

Examining various scissor linkages in the literature, we see that the assemblies comprise either kite (deltoid) or parallelogram loops, or rhombus loops as a more special case (Fig. 3). A kite is a quadrilateral with a pair of short adjacent equal sides and a pair of long adjacent equal sides. A parallelogram also comprises two pairs of equal sides, but equal sides are positioned opposite to each other. A rhombus is an equilateral quadrilateral, which can be considered as an equilateral kite or an equilateral parallelogram.

a)

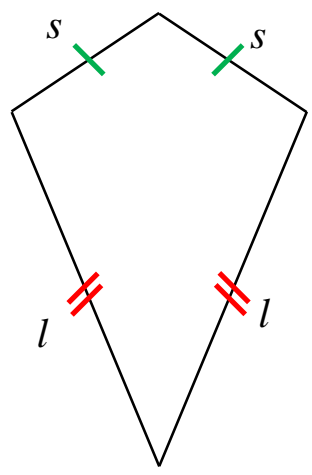

b)

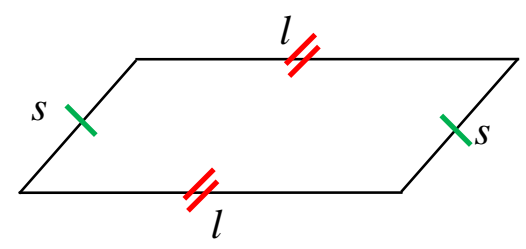

c)

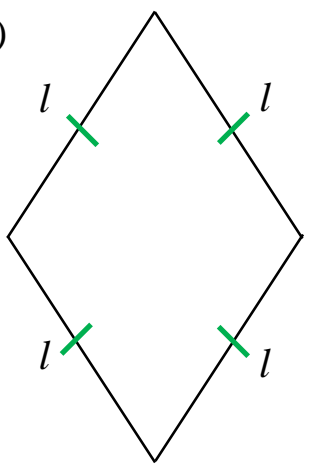

Figure 3 a) Kite, b) Parallelogram, c) Rhombus

Note that both kites and parallelograms comprise only two different side lengths whereas an arbitrary quadrilateral has four different side lengths. Arbitrary quadrilaterals are issued by Bai et al. [35], however such loops are rarely seen in applications. The special constraint on the side lengths result in a certain level of symmetry in designing deployable structures. At this point, it is natural to ask whether there are other quadrilaterals which comprise two short sides of length $s$ and two long sides of length $l$. The short and long lengths can either be adjacent or opposite to each other. However, the convexity of the loop is important as well. The kite and parallelogram loops in Fig. 3 
are convex, but there are also concave versions of them. A concave kite (Fig. 4a) is also called a dart or an arrowhead, while the concave version of a parallelogram is called an anti-parallelogram (Fig. 4b), also called a contra-parallelogram or a crossed parallelogram [43]. Unlike kites and parallelograms, the loop area becomes zero when all link lengths of a dart or an anti-parallelogram are equated.

a)

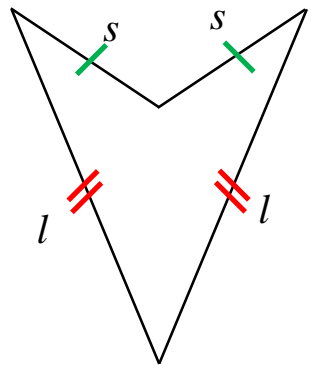

b)

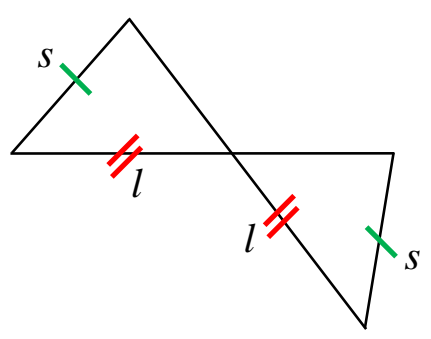

Figure 4 a) Dart, b) Anti-parallelogram

Considering these quadrilaterals as loops of linkages, a kite loop and a dart loop are different assembly modes of the same loop of a linkage. During the motion of the linkage, in order to change the assembly mode of a kite loop into a dart loop without disassembling the loop or vice-versa, the loop should pass through a singular configuration where the two short links are inline (Fig. 5). For example, the kite loops in the assemblies illustrated in Fig. 5 of [36] turn into dart loops during the motion of the linkage.
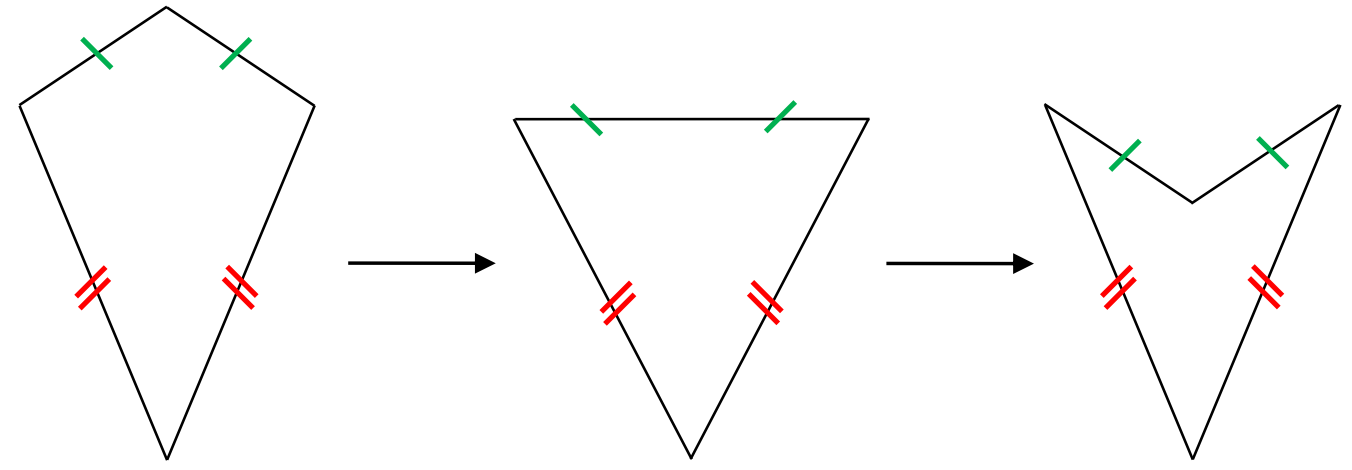

Figure 5 Assembly mode change of a kite loop into a dart loop through the singular configuration

Similarly, a parallelogram and an anti-parallelogram would be the two assembly modes of a loop of a linkage. During the motion of the linkage, a parallelogram loop may change into an anti-parallelogram loop if the loop passes through the singular configuration where all links become collinear. In Fig. 6, both short links move counter-clockwise until the singular configuration is reached. After this singular configuration, if the left link rotated clockwise, the right link may rotate counter-clockwise, hence taking the form of an anti-parallelogram.

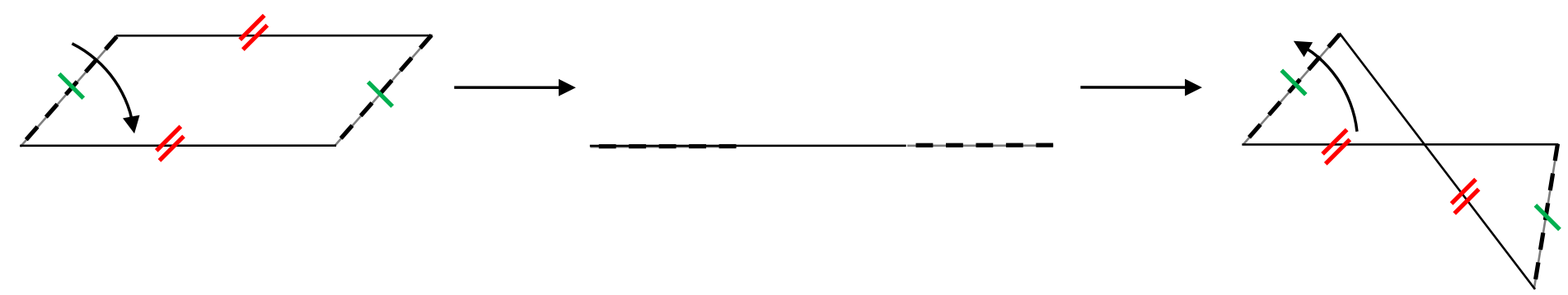

Figure 6 Assembly mode change of a parallelogram loop into an anti-parallelogram loop through the singular configuration 
In a multi-loop linkage, the loops may constrain each other such that some or neither of the loops pass through singular configurations, and hence assembly mode change does not occur. Also, assembly mode change may not be possible due to link collisions. A parallelogram loop is less likely to go through assembly mode change, because the mode change requires all links to be collinear and this results in link collisions unless there is a special constructional design. Therefore, the loop assemblies of rhombuses, kites, darts, parallelograms and antiparallelograms result in different mechanisms with different motion characteristics. The next section is devoted to systematically list all possible loop assemblies composed of the five mentioned loop types.

\section{Loop Assemblies}

A deployable or transformable linkage can be obtained by assembling several loops at their vertices. When two loops are assembled at a common vertex, two pairs of adjacent sides are rigidly connected to each other to constitute a pair of links hinged at the common vertex. For instance, for the assembly of two rhombus loops illustrated in Fig. 7a, the adjacent sides of the loops can be connected to each other to obtain two possible types of Watt-type 6-link kinematic chains. In one of the chains, lower side of left loop is connected to the upper side of the right loop and vice versa (Fig. 7b). Due to the resulting shape this connection type shall be named as X-type connection. In the other alternative chain, upper and lower sides of left and right loops are connected to each other (Fig. 7c). Due to the resulting shape this connection shall be named as V-type connection. Typically, X-type connections are used in scissor linkages, but V-type connections are also seen in certain linkages (see for ex. [44]).

a)

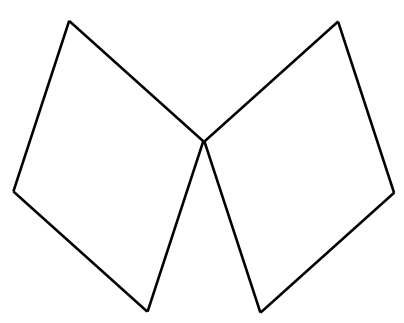

b)

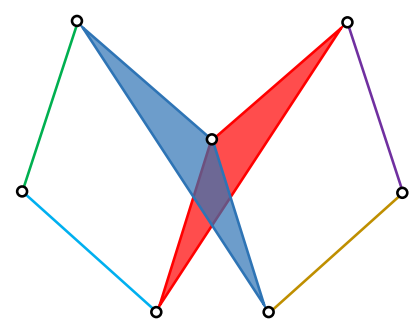

c)

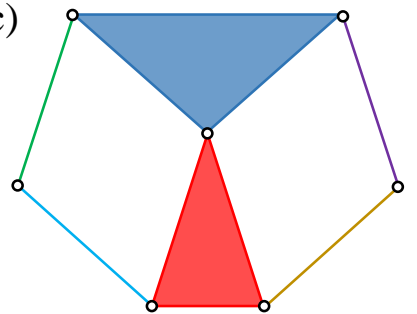

Figure 7 a) Assembly of two rhombus loops and b, c) 6-link kinematic chains obtained from this assembly by rigidly connecting two pairs of adjacent sides of the loops

In practice, mostly, identical loops are used in an assembly, but different kinds of loops and/or loops of different size can also be used. For example, Hoberman [13] used different sizes of rhombi for the Hoberman Ball. An example for the use of combinations of different loops was given by Bouleau and Guscetti [31] where kite and parallelogram loops are used, whereas Zhang et al. [30] used rhombus loops in combination with kite and dart loops. However, the design approach in these latter two papers are unit-based designs, not loop-based designs. In this paper, we shall work on possible assemblies of identical type of loops, but not necessarily of the same size.

For assembling loops, we consider a series of loops juxtaposed along a curve, which are discretized into line segments. For a systematic classification of ways of assembling loops, we shall consider patterns along a line. Afterwards, the line can be dissected into line segments representing a discretized version of a planar curve.

Patterns along a line are called frieze patterns and there are seven distinct such patterns [45]. Frieze patterns are obtained as combination of translation (T) operation with other four basic isometry operations: identity (I), half turn (or $180^{\circ}$ rotation) $(\mathrm{R})$, horizontal reflection about the line $(\mathrm{H})$, vertical reflection about a normal to the line (V) and glide reflection $(\mathrm{G})$ operations (Fig. 8a). To get a frieze pattern, first the translation operation is combined with one or two of the other operations and then the obtained figure is indefinitely replicated along a line. The seven frieze patterns can be listed as TI, TG, TV, TR, TVR, TH and THV. The first four frieze patterns for a general quadrilateral loop is depicted in Fig. 8b. In these patterns, two of the opposite corners of a loop are placed on the line. Unlike the other five patterns, TVR and THV patterns are obtained by repetition of a figure with four copies of the original shape, hence they will not be used in loop patterns. Also, horizontal reflection operation results in overlapping loops, which is not desirable, hence TH and THV patterns will not be used in loop patterns. Therefore, only the first four frieze patterns will be used. 
Besides the frieze patterns, an alternative way to obtain patterns of quadrilateral loops on a curve is by connecting long and short diagonals of the loop, which we shall name as long/short diagonal (LS) patterns. These types of connections can be seen in [35] for rhombus, kite, and parallelogram loops. In general, four such possible patterns of quadrilateral loops can be obtained by rotating the loop clockwise (C) or counter-clockwise (CC) or combining the rotation with a horizontal reflection. Amount of rotation depends on the angle between the diagonals of the loops. Accompanied with a translation, the four possible patterns can be listed as TC, TCC, TCH and TCCH patterns as depicted in Fig. 8c.

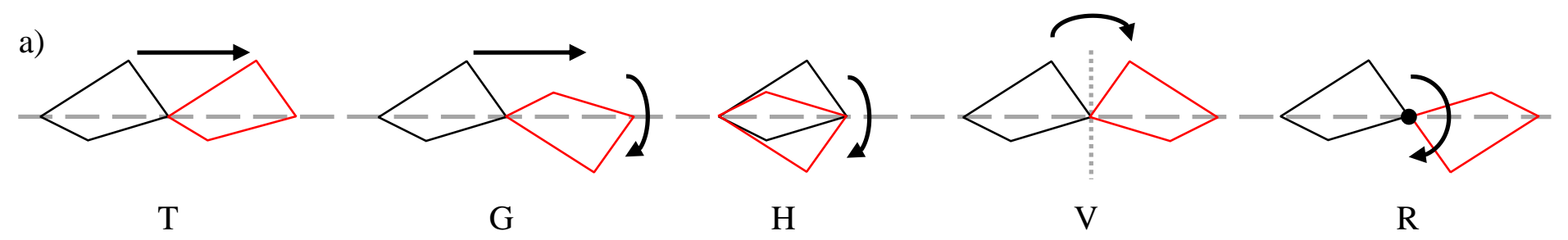

b)

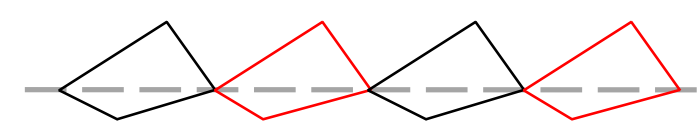

TI

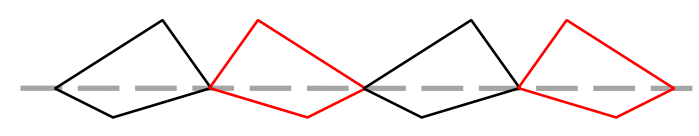

TV

c)

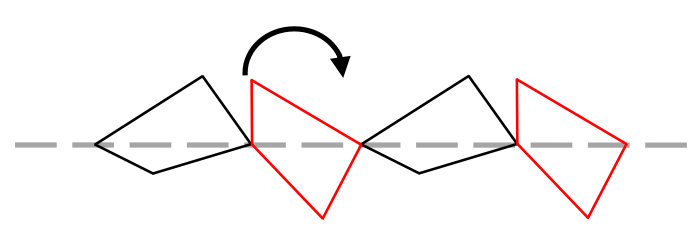

TC

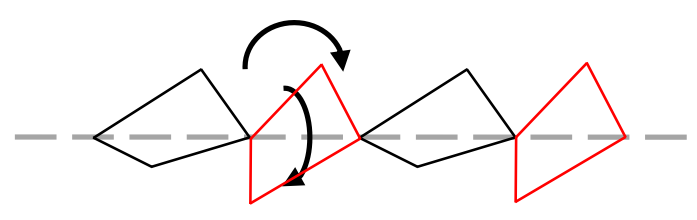

$\mathrm{TCH}$

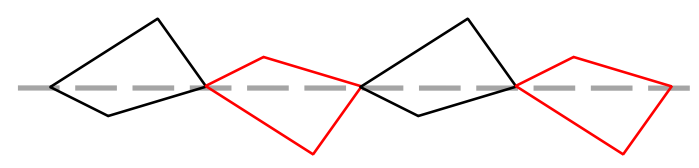

TG

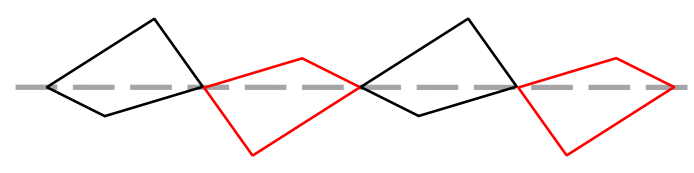

TR

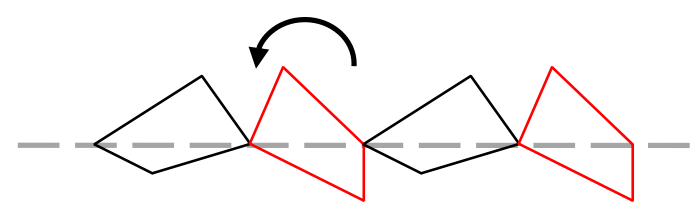

TCC

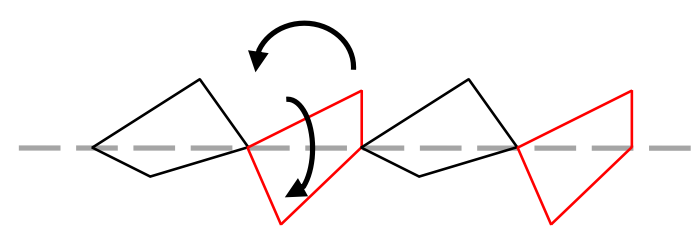

$\mathrm{TCCH}$

Figure 8 a) Basic isometry operations, b) the first four frieze patterns, c) long-short diagonal connections

The eight patterns presented in Fig. 8b-c are applied to the five basic loops (rhombus, kite, dart, parallelogram, anti-parallelogram) in order to obtain possible loop assemblies. Since each quadrilateral loop under consideration possess some symmetries, some of the eight patterns turn out to be identical. Once the patterns are obtained, Xtype and V-type connection options can be evaluated in order to determine the link geometries. The loop assemblies with X-type connections are listed in the forthcoming discussions in this section. Each pattern also has a $\mathrm{V}$-type connection, but those assemblies result in a motion such that as a diagonal of a loop expands, the diagonals 
of adjacent loops contract, so the resulting motions are generally not favorable. Therefore, the loop assemblies with V-type connections are not classified in this study, but only some examples are presented at the end of the section.

A rhombus loop has horizontal and vertical mirror symmetry, so all four frieze patterns result in the same pattern. In addition, all LS patterns result in the same pattern. Table 1 lists the possible two patterns on a line and examples of loop assemblies with X-type connections on a circular arc. In Table 1, the patterns are depicted on a straight line for clarity, but in general the patterns can be on any arbitrary curve resulting in a scaling deployable linkage. As it is well known since Hoberman's patent [13], the rhombus loop assemblies with TI pattern can be used for scaling of any curve. In this pattern, all angulated elements are identical. The TC pattern comprises equilateral GAEs and also results in scaling deployment. These scaling assemblies were worked out by Bai et al. [35].

Table 1. Rhombus Loop Assemblies

\begin{tabular}{|c|c|c|c|}
\hline $\begin{array}{l}\text { Pattern } \\
\text { Type }\end{array}$ & Pattern & Linkage & $\begin{array}{l}\text { Motion } \\
\text { Type }\end{array}$ \\
\hline $\begin{array}{c}\text { TI } \\
\text { TG } \\
\text { TV } \\
\text { TR }\end{array}$ & & & $\begin{array}{c}\text { Scaling } \\
\text { Deployable }\end{array}$ \\
\hline $\begin{array}{c}\text { TC } \\
\text { TCC } \\
\text { TCH } \\
\text { TCCH }\end{array}$ & & & $\begin{array}{c}\text { Scaling } \\
\text { Deployable }\end{array}$ \\
\hline
\end{tabular}

Kite loop assemblies can be investigated in two distinct subgroups: vertical and horizontal kite loop assemblies. Since a vertical kite has vertical mirror symmetry, TI and TV patterns are identical. TG and TR patterns are also identical due to vertical mirror symmetry (Table 2). The assemblies obtained from TI patterns with X-type connections go through a transformable motion. When the pattern is constructed on a straight line, the assembly can bend upwards and downwards such that the curve can switch from convex to concave from and vice versa. Such transformable assemblies were issued by Yar et al. [36]. A special case is obtained when the pattern is constructed on a circular arc with straight (not angulated) elements, in which case the linkage has angular deployable motion (Table 3). 
Table 2. Vertical Kite Loop Assemblies

\begin{tabular}{|c|c|c|c|}
\hline $\begin{array}{c}\text { Pattern } \\
\text { Type }\end{array}$ & Pattern & Linkage & Motion Type \\
\hline $\begin{array}{l}\text { TI } \\
\text { TV }\end{array}$ & & . & $\begin{array}{c}\text { Transformable } \\
\text { (concave/convex) } \\
\text { (conves }\end{array}$ \\
\hline $\begin{array}{l}\text { TG } \\
\text { TR }\end{array}$ & & & $\begin{array}{c}\text { Scaling } \\
\text { Deployable }\end{array}$ \\
\hline
\end{tabular}




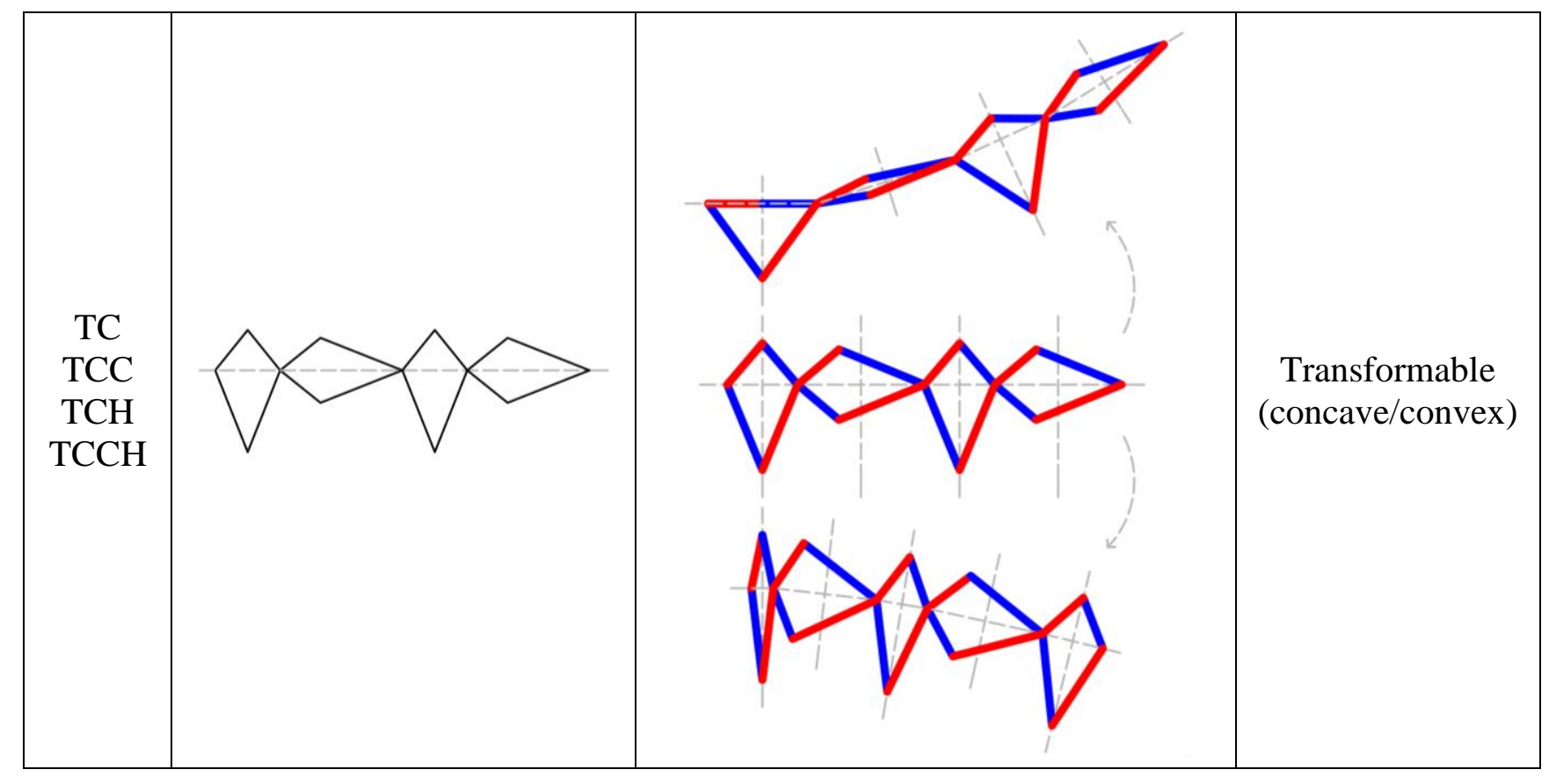

Table 3. Special case: Vertical Kite Loop Assembly on a Circular Arc

\begin{tabular}{|c|c|c|c|}
\hline $\begin{array}{c}\text { Pattern } \\
\text { Type }\end{array}$ & Pattern & Linkage & Motion Type \\
\hline & & & \\
TI & & & \\
\hline
\end{tabular}

The TG pattern of a vertical kite loop results in a linkage with scaling deployment as also noted by Bai et al. [35]. The TC and TCC patterns of a vertical kite loop are mirror images of each other, hence they are not considered as separate cases. Due to horizontal symmetry of the horizontal kite (rotated version of vertical kite), TCH and TCCH patterns are identical with TC and TCC patterns, respectively. The LS patterns result in a transformable linkage with variable curvature.

A horizontal kite has horizontal mirror symmetry, so TI and TG patterns are identical. TV and TR patterns are also identical due to horizontal mirror symmetry (Table 4). The linkages obtained from TI pattern result in a 
transformable motion, whereas the linkages obtained from TV pattern result in scaling deployment. These scaling deployable assemblies were also examined by Bai et al. [35]. LS patterns are already examined in Table 2.

Vertical dart has vertical mirror symmetry, so TI and TV patterns are identical and also TG and TR patterns are identical (Table 5). Just like the vertical kite loop assemblies the assemblies obtained from TI patterns with X-type connections go through a transformable motion as noted by Yar et al. [36] and the TG pattern of a vertical dart loop results in a linkage with scaling deployment. To the best knowledge of the authors, the scaling linkages obtained from TG patterns of vertical dart loops are not noted in the literature before. The long-short diagonal connections of dart loops are kept out of scope in this study, because such assemblies result in too much link collisions.

Table 4. Horizontal Kite Loop Assemblies

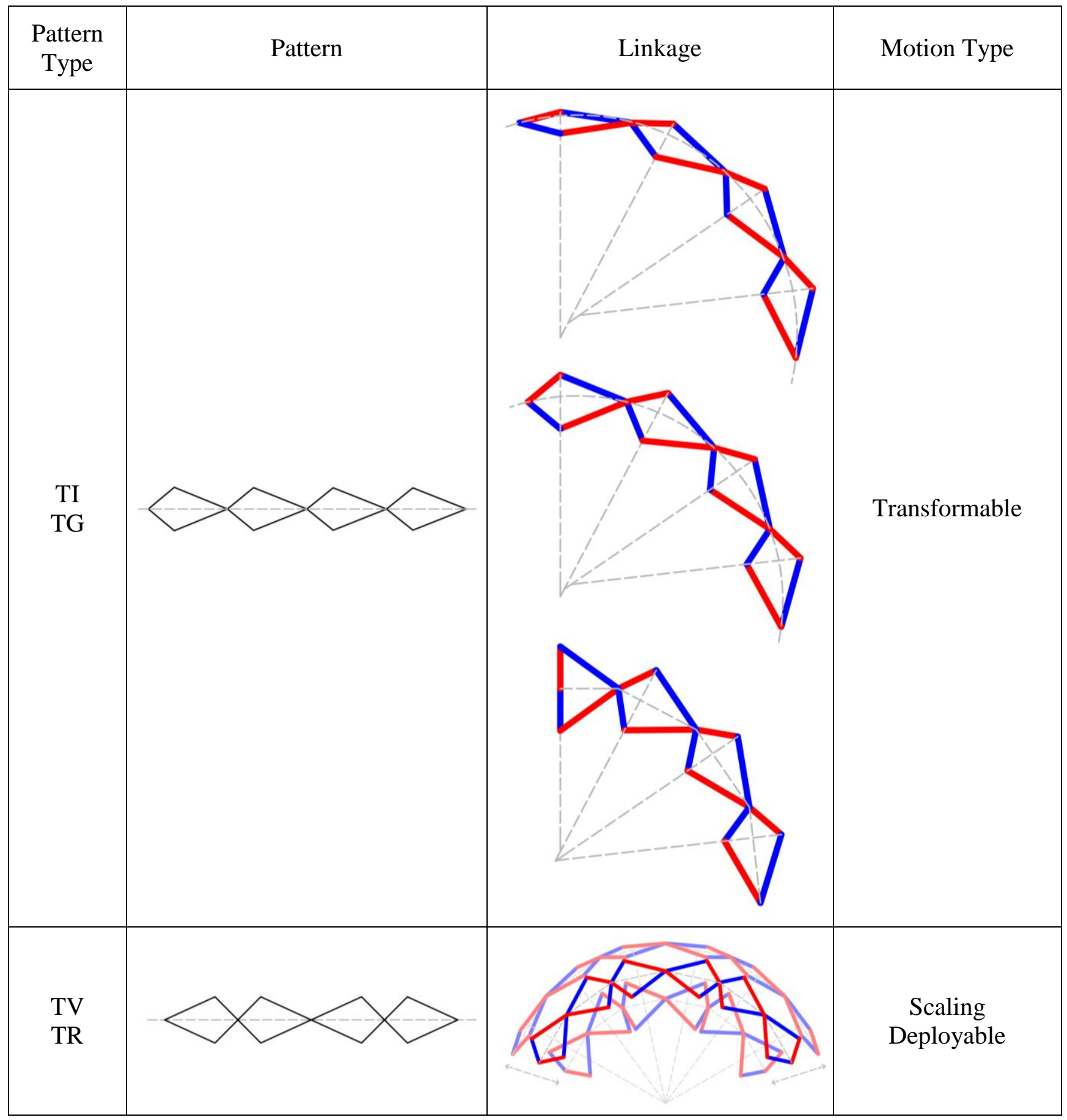


Table 5. Vertical Dart Loop Assemblies

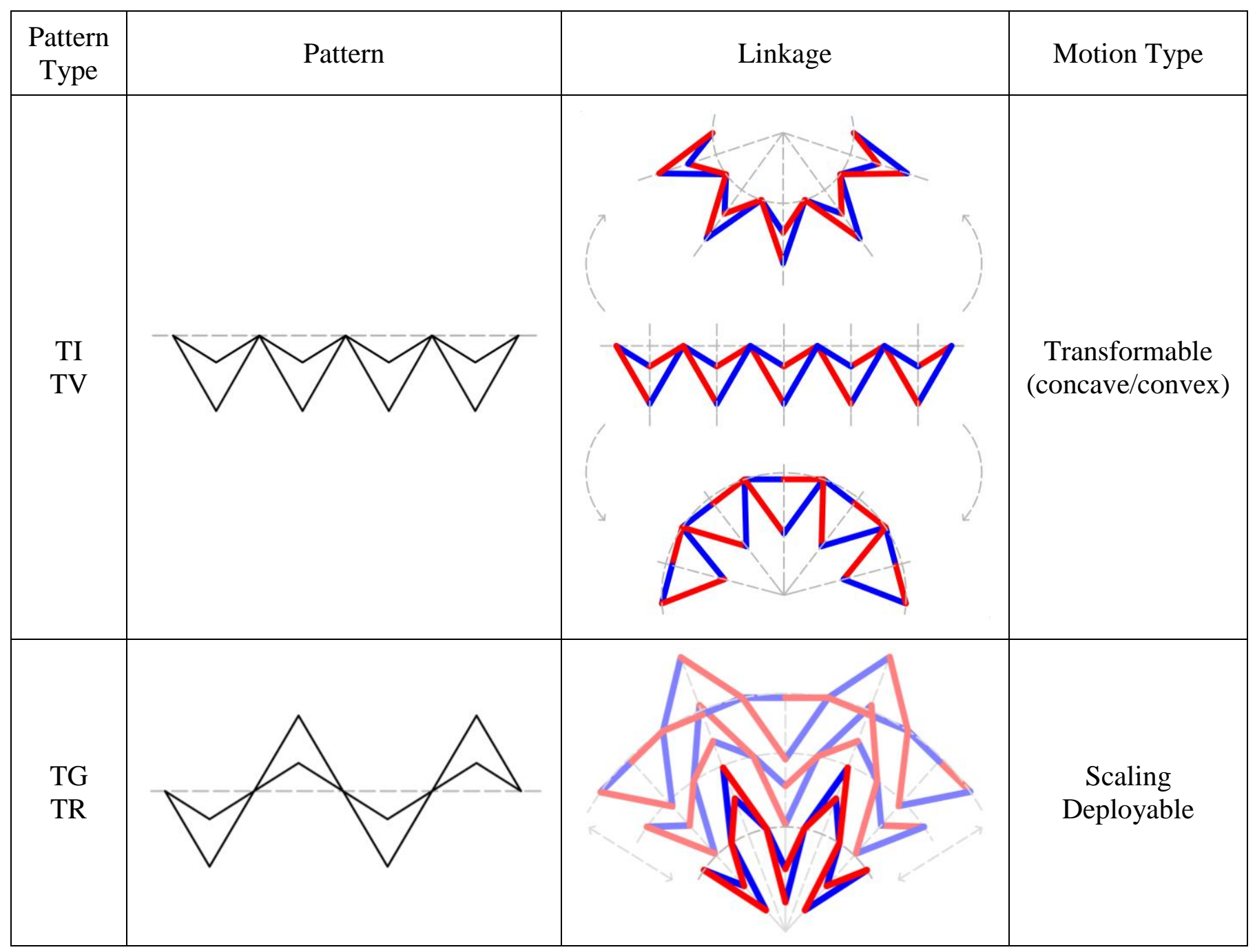

Similar to a horizontal kite, a horizontal dart has horizontal mirror symmetry, so TI and TG patterns are identical (Table 6). TV and TR patterns do not make sense because of too much link collisions, so they are not listed in the table. Linkages obtained from TI pattern result in a transformable motion. 
Table 6. Horizontal Dart Loop Assemblies

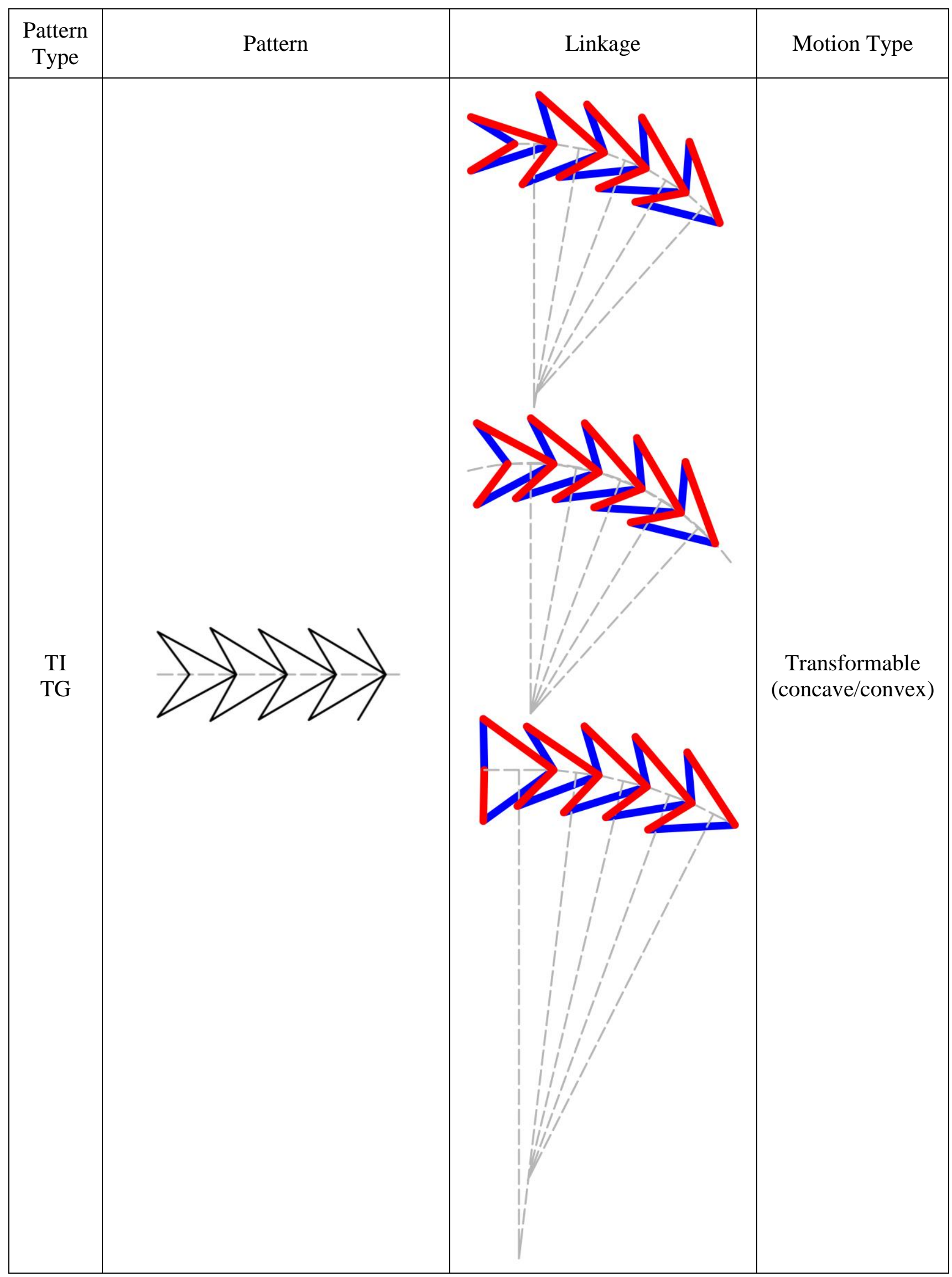


A parallelogram does not possess neither vertical nor horizontal mirror symmetry, but it has a cyclic symmetry of order two, i.e. it has the same shape after half turns. Therefore, the following pairs of patterns are identical: TI and TR patterns; TG and TV patterns; TC and TCCH patterns; TCC and TCH patterns (Table 7).

Table 7. Parallelogram Loop Assemblies

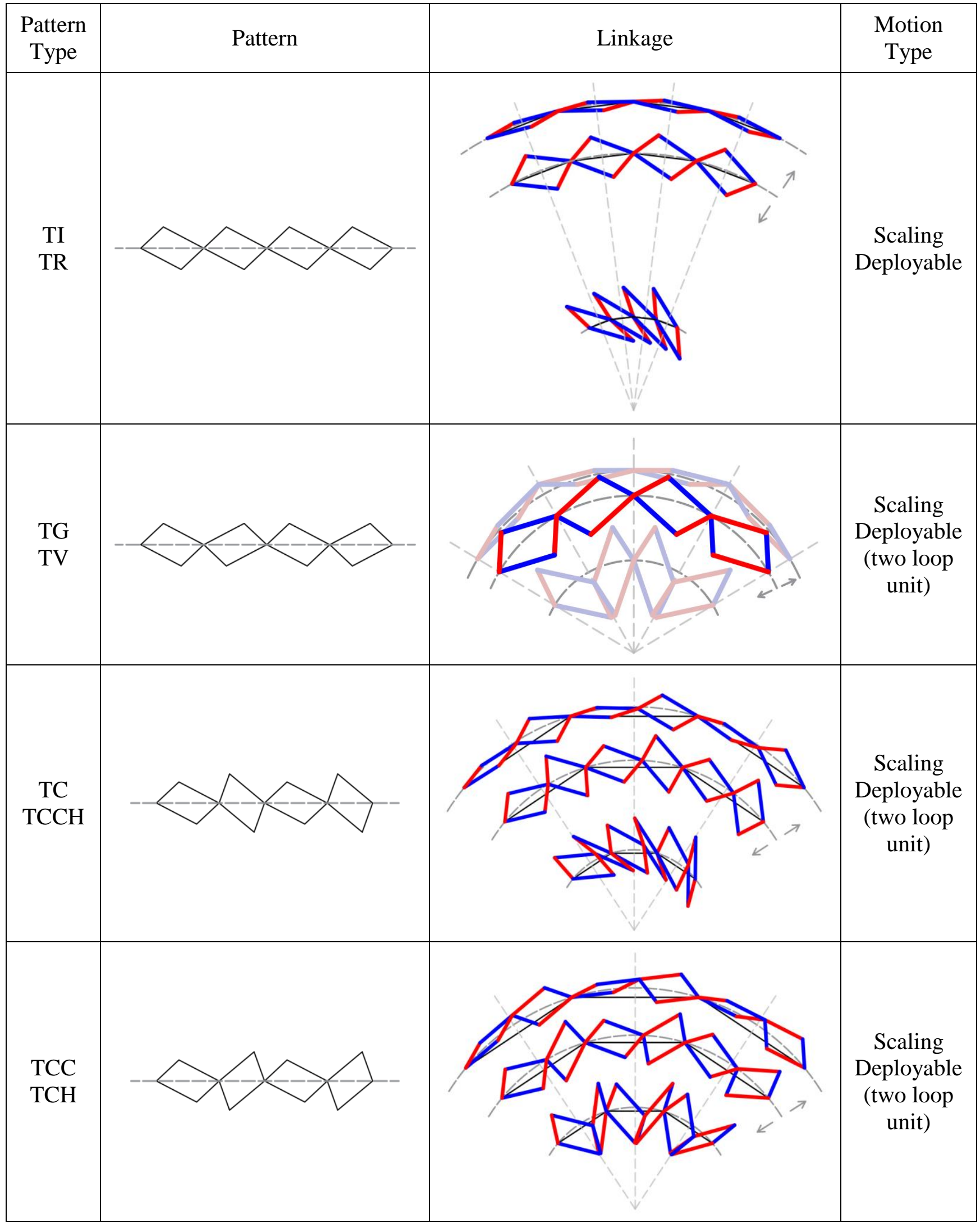


TI patterns with X-type connections go through a scaling-deployable motion as also noted by Bai et al. [35]. The TG, TC and TCC patterns with X-type connections result in a scaling deployable motion, but if only a unit is taken as a pair of consecutive loops. For a TG pattern, the middle hinge in a two-loop unit remains on the normal line of the corresponding line segment, but it does not remain on the line segment. For TC and TCC patterns, the short and long diagonals of two adjacent loops change with different rates and the middle hinge in a two-loop unit neither remains on the corresponding line segment, nor the normal line. To the best knowledge of the authors, the scaling motion of the TG, TC and TCC patterns of parallelogram loop assemblies are not noted in the literature before.

Anti-parallelogram loop assemblies were studied by Gür et al. [37] in detail. An anti-parallelogram loop possesses vertical mirror symmetry, hence the following pairs of patterns are identical: TI and TV patterns; TG and TR patterns; TC and TCC patterns; TCH and TCCH patterns. TI pattern with X-type connections have a transformable motion whereas TG patterns result in scaling deployable motion as issued by Gür et al. [37]. TG patterns with Vtype connections might be considered as special interest and they result in a transformable motion. TC and TCC patterns with X-type connections also result in transformable motion.

Table 8. Anti-parallelogram Loop Assemblies

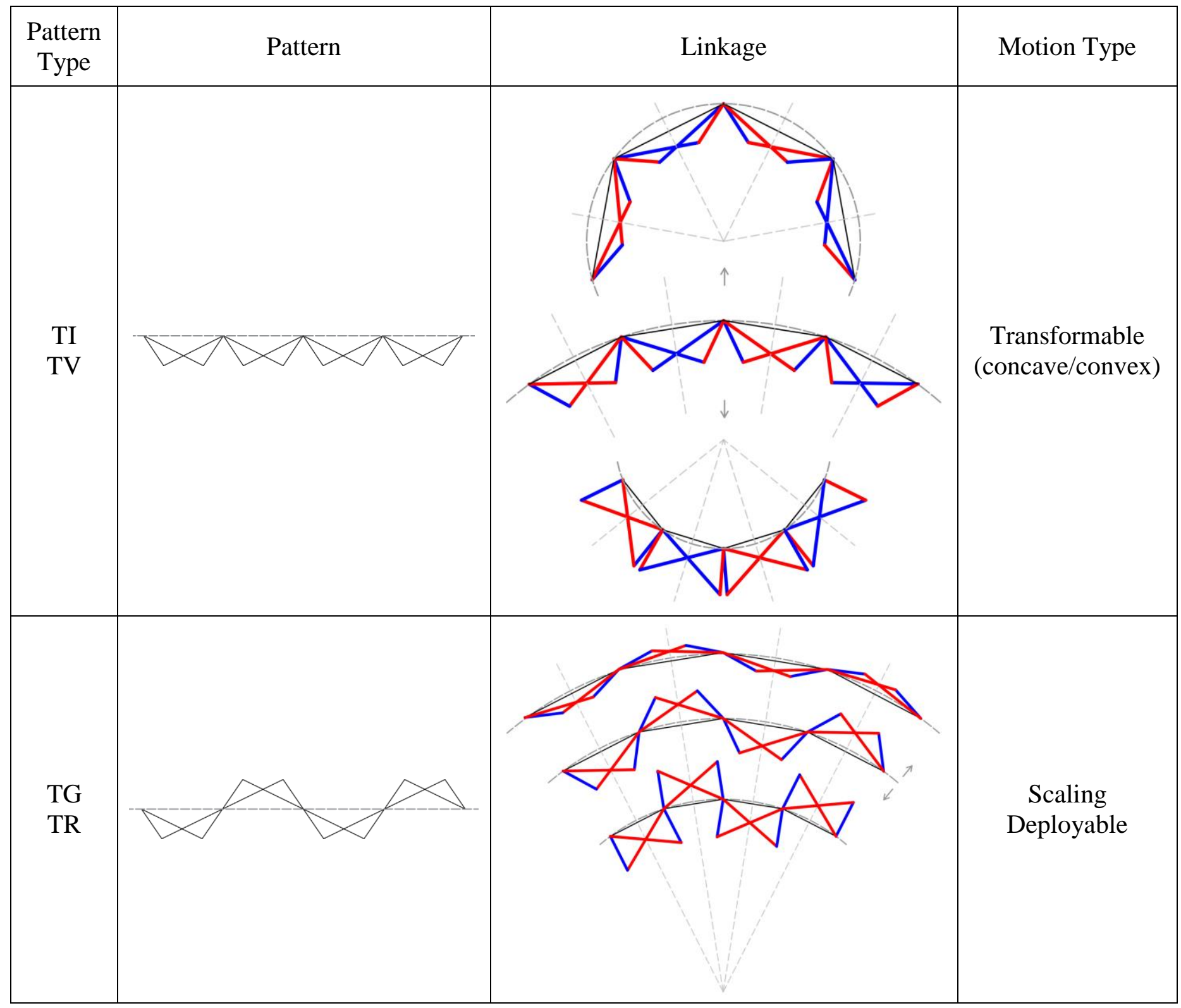




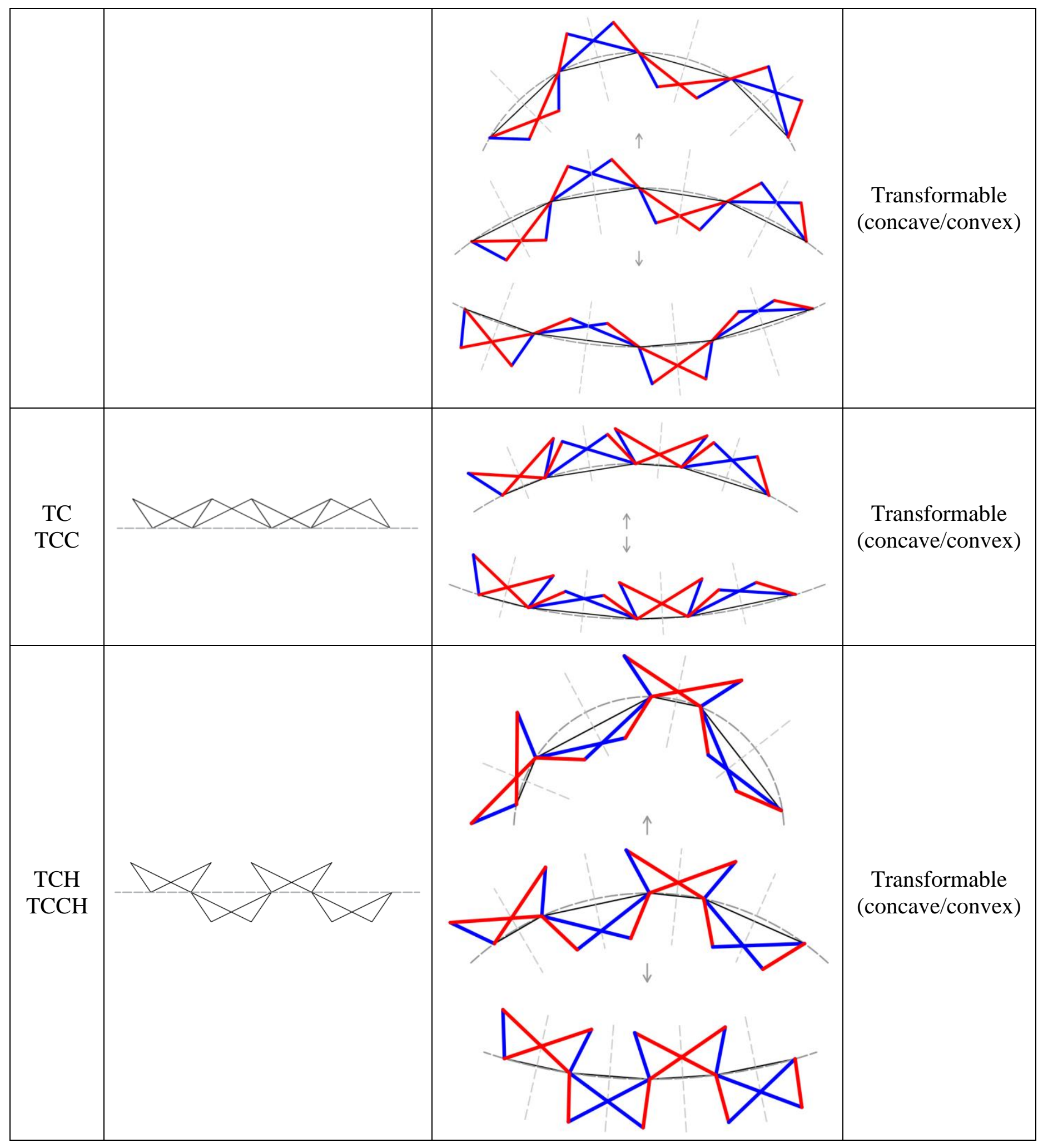

All possible assemblies with V-type connections are also investigated, but the resulting motions are generally not found to be of practical importance, as specified at the beginning of the section. Besides the TG pattern of the antiparallelograms depicted in Table 8, one of the rare cases of interest with V-type connections is presented in Figs. 9 and 10, where the rhombus loops are assembled in a circle (Figs. 9b and 10b) with TI pattern in order to obtain hexagonal and orthogonal assemblies which are capable of scaling deployment. After the assembly, the outer links are straight rods (angulated elements with $180^{\circ} \mathrm{kink}$ angle) and the inner links are isosceles angulated elements. It 
can be seen from the motion that a pair of opposite joints in each rhombus loop move on fixed straight lines, which suggests that all links have the double-slider motion, that is the Cardan motion (see [46]).

a)

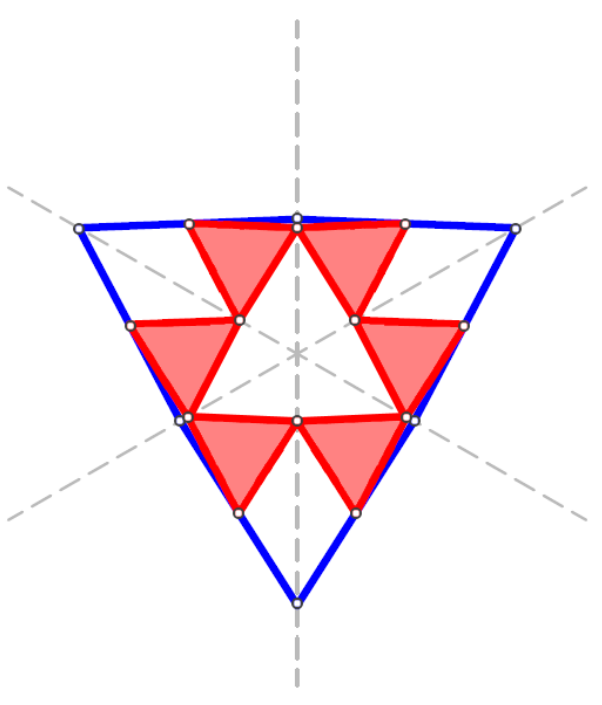

b)

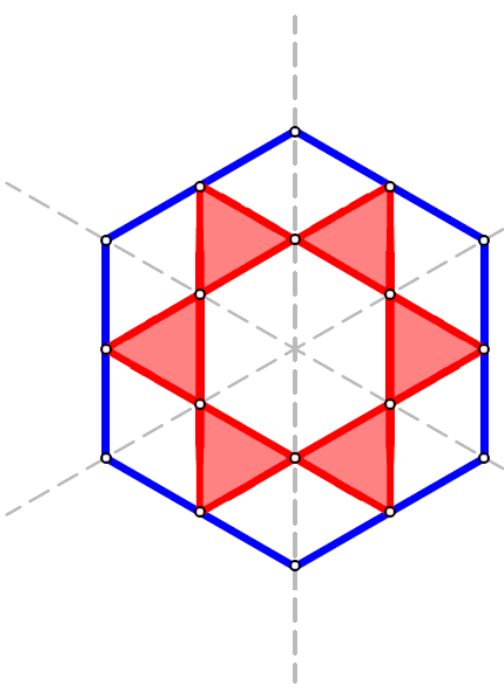

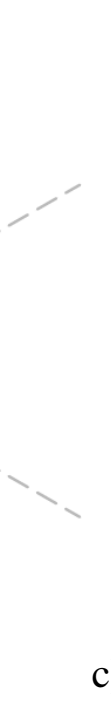

c)

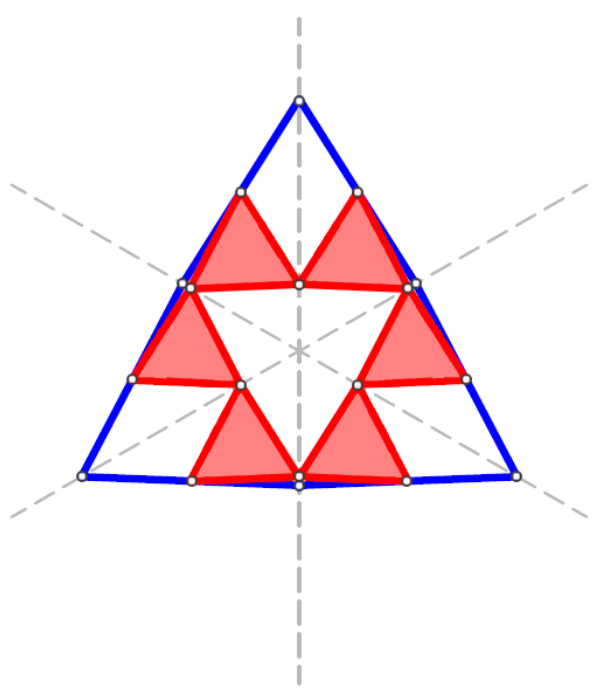

d)

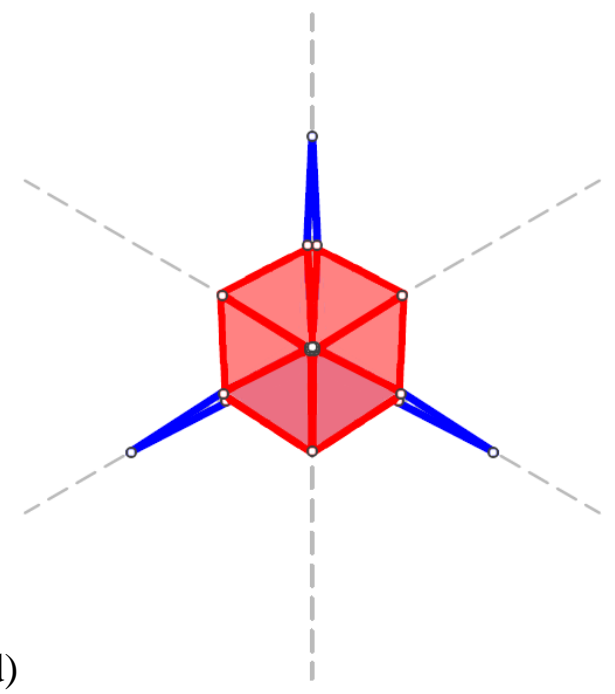

e)

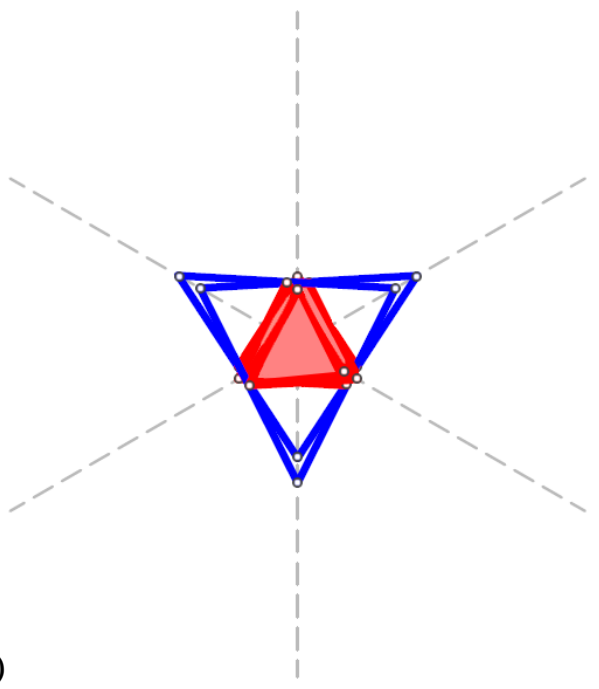

Figure 95 configurations of a hexagonal closed loop rhombus assembly with V-type connections and TI pattern 
a)

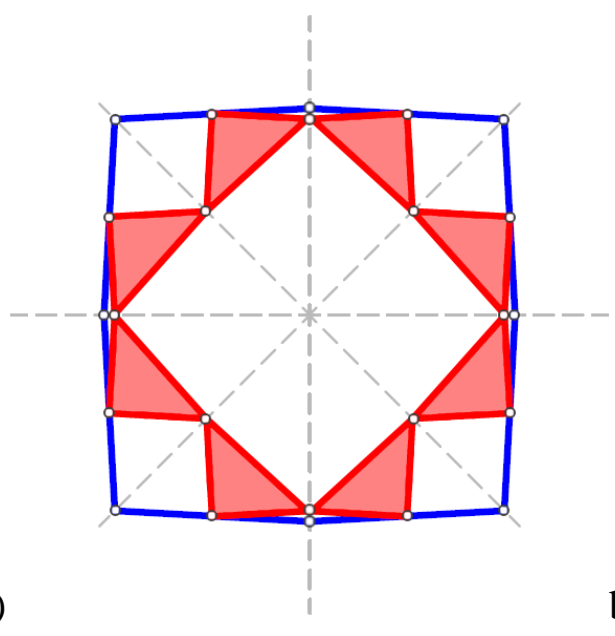

b)

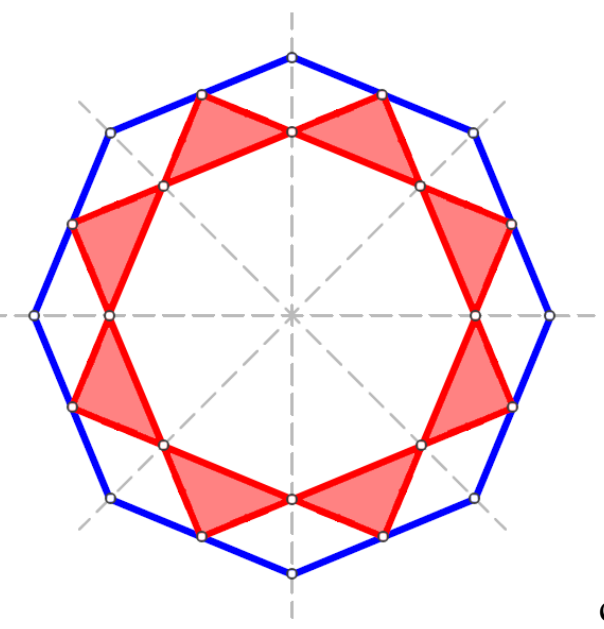

c)

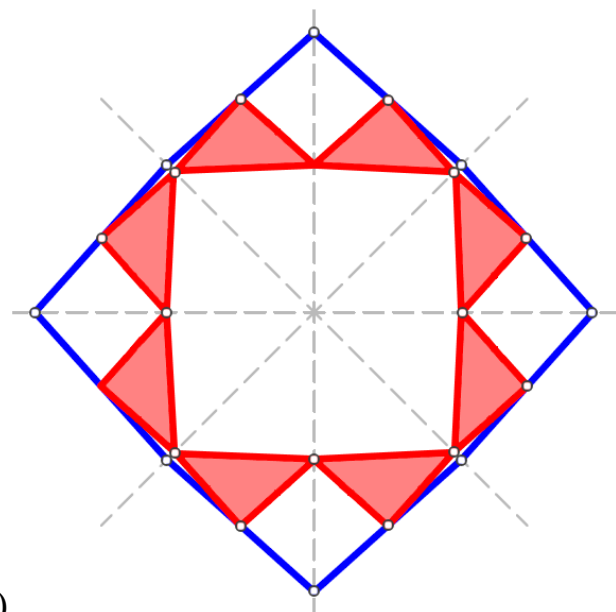

e)

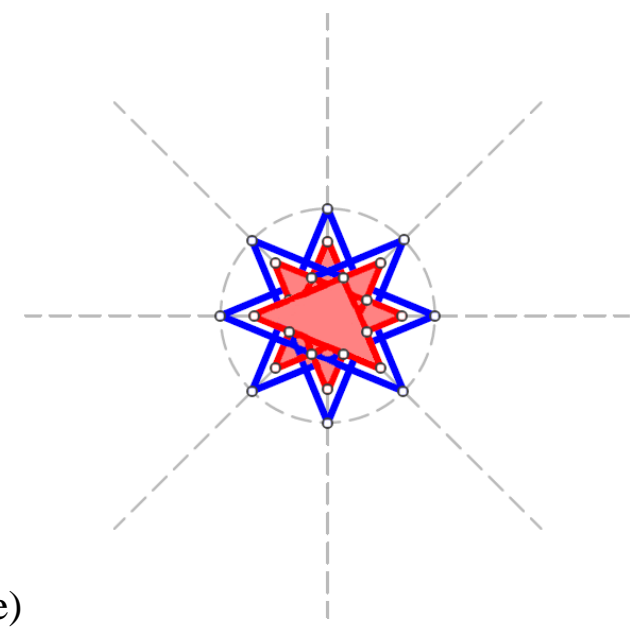

d)

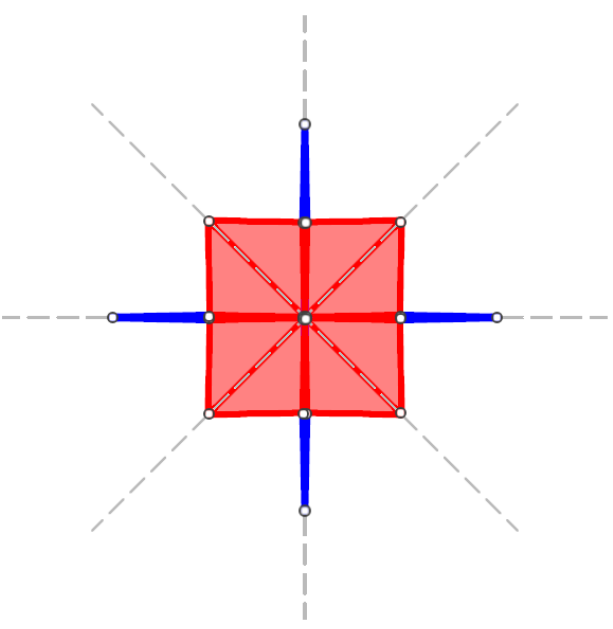

Figure 105 configurations of an octagonal closed loop rhombus assembly with V-type connections and TI pattern

\section{Conclusions}

This study presents a systematic way to list possible scissor linkages obtained by assembling rhombus, kite, dart, parallelogram and anti-parallelogram loops using frieze patterns and long-short diagonal connections. For the dart and kite loops, assemblies of vertical and horizontal loops are evaluated separately. For each obtained linkage, the motion characteristics is specified as being scaling deployable, angular deployable or transformable. The linkages listed in this study may be used as a library of scissor linkage topologies. A summary of the results for X-type connections may be seen in Table 9, where whether a scalable deployable motion or a transformable motion is obtained for the assemblies of a given loop type with a given pattern. Since angular deployment is only obtained for TI pattern of vertical kite loop assembly on a circular arc, it is not presented in Table 9. In Table 9, S stands for scalable deployable and T stands for transformable. Merged adjacent cells and also cells with the same superscript $(\mathrm{a}, \mathrm{b}, \ldots \mathrm{h})$ correspond to the same assembly. Accordingly, 10 distinct scalable deployable and 8 distinct transformable assemblies are listed.

Most of the obtained linkages already exist in the literature, but some novel linkages are also obtained. Since scaling linkages are of great importance, especially the vertical dart loop assembly obtained from TG patterns and parallelogram loop assemblies obtained from TG, TC and TCC patterns may be specified as important novel linkages presented in this study.

Dimensional synthesis of these scissor linkages as a general formulation or for specific tasks may be issued in future studies. The novel linkages have potential applications in kinetic architecture, outer-space applications, furniture design and machinery. 
Table 9. Summary of loop assemblies with X-type connections and their motion types

\begin{tabular}{|c|c|c|c|c|c|c|c|c|}
\hline Loop Pattern & TI & TG & TV & TR & $\mathrm{TC}$ & TCC & $\mathrm{TCH}$ & $\mathrm{TCCH}$ \\
\hline Rhombus & \multicolumn{4}{|c|}{$\mathrm{S}$} & \multicolumn{4}{|c|}{$\mathrm{S}$} \\
\hline Vertical kite & $\mathrm{T}^{\mathrm{a}}$ & $S^{b}$ & $\mathrm{~T}^{\mathrm{a}}$ & $S^{b}$ & \multirow{2}{*}{\multicolumn{4}{|c|}{$\mathrm{T}$}} \\
\hline Horizontal kite & \multicolumn{2}{|c|}{$\mathrm{T}$} & \multicolumn{2}{|c|}{$\mathrm{S}$} & & & & \\
\hline Vertical dart & $\mathrm{T}^{\mathrm{c}}$ & $S^{d}$ & $\mathrm{~T}^{\mathrm{c}}$ & $S^{d}$ & \multirow{2}{*}{\multicolumn{4}{|c|}{$\begin{array}{c}\text { Not practical due to link } \\
\text { collisions }\end{array}$}} \\
\hline Horizontal dart & \multicolumn{2}{|c|}{$\mathrm{T}$} & & & & & & \\
\hline Parallelogram & $\mathrm{S}^{\mathrm{e}}$ & \multicolumn{2}{|c|}{$\mathrm{S}$} & $\mathrm{S}^{\mathrm{e}}$ & $S^{f}$ & \multicolumn{2}{|c|}{$\mathrm{S}$} & $S^{f}$ \\
\hline Anti-parallelogram & $\mathrm{T}^{\mathrm{g}}$ & $\mathrm{S}^{\mathrm{h}}$ & $\mathrm{T}^{\mathrm{g}}$ & $S^{h}$ & \multicolumn{2}{|c|}{$\mathrm{T}$} & \multicolumn{2}{|c|}{$\mathrm{T}$} \\
\hline
\end{tabular}

\section{Acknowledgements}

This project has received funding from the European Union's Horizon 2020 research and innovation programme under the Marie Skłodowska-Curie grant agreement No 689983.

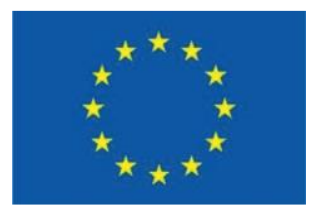

\section{References}

[1] True M, Hamma K (eds) 1994 A Passion for Antiquities: Ancient Art from the Collection of Barbara and Lawrence Fleischman. Exhibition Catalog, Malibu, The J. Paul Getty Museum, pp. 320-323

[2] Piñero EP 1961 Project for a mobile theatre. Archit. Design. 12: 154-155

[3] Piñero EP 1962 Expandable space framing. Prog. Arch. 43: 154-155

[4] Piñero EP 1965 Three Dimensional Reticular Structure. Patent no: US3185164

[5] Zeigler T 1976 Collapsable Self-Supporting Structures. Patent no: US3968808

[6] Clarke RC 1984 The kinematics of a novel deployable space structure system. In: Nooshin H (ed) Proc. 3rd International Conference on Space Structures. Guildford, Elsevier Applied Science Publishers, pp. 820-822

[7] Escrig F 1984 Expandable space frame structures. In: Nooshin H (ed), Proc. 3rd International Conference on Space Structures. Guildford, Elsevier Applied Science Publishers, pp. 845-850

[8] Escrig F 1985 Expandable space structures. Int. J. Space Struct. 1: 79-91

[9] Escrig F, Valcárcel JP 1987 Curved expandable space grids. In: Proc. International Conference on the Design and Construction of Non-Conventional Structures. London, pp. 157-166

[10] Escrig F, Valcárcel JP 1993 Geometry of expandable space structures. Int. J. Space Struct. 8: 71-84

[11] F. Escrig and J. Sanchez 2006 New designs and geometries of deployable scissor structures. In: Adaptables2006, International Conference on Adaptive Building Structures. Eindhoven, TU/e, pp. 18-22

[12] Escrig F 1996 General survey of deployability in architecture. In: Proc. MARAS'96: 2nd International Conference on Mobile and Rapidly Assembled Structures. Seville, Computational Mechanics Publications, pp. 3-22

[13] Hoberman C 1990 Reversibly Expandable Doubly-curved Truss Structure. Patent no: US4942700

[14] Hoberman C 1991 Radial Expansion/Retraction Truss Structures. Patent no: US5024031

[15] You Z, Pellegrino S 1997 Foldable bar structures. Int. J. Solids Struct. 34: 1825-1847

[16] Kassabian PE, You Z, Pellegrino S 1999 Retractable roof structures. P. I. Civil Eng. - Str. B. 134: 45-56

[17] Al Khayer M, Lalvani H 1998 Scissors-action deployables based on space-filling of polygonal hyperboloids. In: Pellegrino S, Guest SD (eds), IUTAM-IASS Symposium on Deployable Structures: Theory and Applications. Dordrecht, Kluwer Academic Publishers, pp. $1-10$ 
[18] Gantes CJ 1996 Analytical predictions of the snap-trough characteristics of deployable structures. In: Proc. MARAS '96: Second International Conference on Mobile and Rapidly Assembled Structures. Seville, Computational Mechanics Publications, pp. 83-92

[19] Gantes CJ, Logcher RD, Connor JJ, Rosenfeld Y 1993 Deployability conditions for curved and flat, polygonal and trapezoidal deployable structures. Int. J. Space Struct. 8: 97-106

[20] Langbecker T 1999 Kinematic analysis of deployable scissor structures. Int. J. Space Struct. 14: 1-15

[21] Langbecker T, Albermani F 2001 Kinematic and non-linear analysis of foldable barrel vaults. Eng. Struct. 23: 158-171

[22] Kokawa T 1997 Cable scissors arch-marionettic structure. In: Chilton JC, Choo B, Lewis WJ, Popovic O (eds), Structural Morphology: Towards the New Millennium. Nottingham, University of Nottingham. 107-116

[23] Kokawa T 2000 Structural idea of retractable loop-dome. J. Int. Assoc. Shell Spatial Struct. 41: 111-116

[24] Van Mele T 2008 Scissor-Hinged Retractable Membrane Roofs. PhD thesis, Vrije Universiteit Brussel, Brussel

[25] Rippmann M 2008 Curtain Wall: Building Design Semesterarbeit. Stuttgart: Universität Stuttgart-ILEK

[26] Petrova P, Sommer B, 2008 An anti-clastic, double-curved SLEs structure. In: Proc. 1st Symposium on Architectural Geometry, Advances in Architectural Geometry. Vienna, pp. 111-114

[27] Akgün Y 2010 A Novel Transformation Model for Deployable Scissor-hinge Structures. PhD thesis, Izmir Institute of Technology, İzmir

[28] Akgün Y, Gantes CJ, Sobek W, Korkmaz K, Kalochairetis K 2011 A novel adaptive spatial scissor-hinge structural mechanism for convertible roofs. Eng. Struct. 33: 1365-1376

[29] Roovers K, Mira LA, De Temmerman N 2013 From surface to scissor structure. In: Proc. First Conference Transformables 2013. Seville, Starbooks, pp. 275-280

[30] Zhang R, Wang S, Chen X, Ding C, Jiang L, Zhou J, Liu L 2016 Designing planar deployable objects via scissor structures. IEEE Trans Vis Comput Graph. 22: 1051-1062

[31] Bouleau E, Guscetti G 2016 Scissor mechanisms for transformable structures with curved shape: the "Jet d'Eau" movable footbridge in Geneva. In: Adriaenssens S, Gramazio F, Kohler M, Menges A, Pauly M (eds) Advances in Architectural Geometry 2016. Zürich, vdf Hochschulverlag, pp. 222-239

[32] Hoberman C 2013 Mechanical Invention Through Computation - Mechanism Basics. MIT Class 6.S080 Lecture Notes, pp. 37-47

[33] Liao Q, Li D 2005 Mechanisms for scaling planar graphs. Chin. J. Mech. Eng. 41: 140-143

[34] Kiper G, Söylemez E 2010 Irregular polygonal and polyhedral linkages comprising scissor and angulated elements. In: Proc. 1st IFToMM Asian Conference on Mechanism and Machine Science, Taipei, pp. 455-459

[35] Bai G, Wei S, Li D, Liao Q, Kong X 2014 A novel synthesis method of polygon-scaling mechanisms. In: Proc. ASME 2014 International Design Engineering Technical Conferences \& Computers and Information in Engineering Conference. Buffalo, NY. DETC2014-34934

[36] Yar M, Korkmaz K, Kiper G, Maden F, Akgün Y, Aktaş E 2017 A novel planar scissor structure transforming between concave and convex configurations. Int. J. Comput. Methods Exp. Meas. 5: 442-450

[37] Gür Ş, Korkmaz K, Kiper G 2017 Radially expandable ring-like structure with anti-parallelogram loops. In: Proc. International Symposium of Mechanism and Machine Science (ISMMS-2017). Baku, pp. 150-155

[38] Gür Ş, Korkmaz K, Kiper G 2019 Design of anti-parallelogram loop assemblies. J. Int. Assoc. Shell Spatial Struct. 60: 232-240

[39] Karagöz, C. 2018 Kinematic Design of Scissor Linkages. MSc thesis, Izmir Institute of Technology, İzmir

[40] Maden F, Ölmez D, Gür Ş, Uncu MY, Mitropoulou C 2019 Dynamic shelter structure. In: Cruz PJS (ed) Structures and Architecture - Bridging the Gap and Crossing Borders. London, CRC Press, pp. 689-696

[41] Maden F, Korkmaz K, Akgün Y 2011 A review of planar scissor structural mechanisms: geometric principles and design methods. Archit. Sci. Rev. 54: 246-257

[42] Maden F, Akgün Y, Kiper G, Gür Ş, Yar M, Korkmaz K 2019 A critical review on classification and terminology of scissor structures. J. Int. Assoc. Shell Spatial Struct. 60: 47-64

[43] Wikipedia contributors (2021) Antiparallelogram. In: Wikipedia, The Free Encyclopedia. Retrieved October 21, 2021, https://en.wikipedia.org/w/index.php?title=Antiparallelogram\&oldid=1050296211

[44] Wohlart K 2000 Double-chain mechanism. In: Proc. IUTAM-IASS Symposium on Deployable Structures: Theory and Application, pp. 457-466

[45] Conway JH, Burgiel H, Goodman-Strauss C 2008 The seven types of frieze patterns. In: The Symmetries of Things. Wellesley, MA, A K Peters, pp. 67-73

[46] Kiper G, Söylemez E, Kişisel AUÖ 2008 A family of deployable polygons and polyhedra. Mech. Mach. Theory. 43: 627-640 